\title{
Target object moderation of attentional orienting by gazes or arrows
}

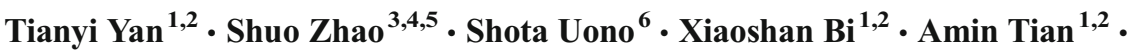 \\ Sayaka Yoshimura $^{6} \cdot$ Motomi Toichi $^{3,5}$
}

Published online: 9 August 2016

(C) The Psychonomic Society, Inc. 2016

\begin{abstract}
Recent studies have attempted to demonstrate the importance of the characteristics of directional cues and observers' traits in attentional orienting. This study investigated how attentional orienting is influenced by target processing. Two experiments showed the critical role played by target processing in attentional orienting that relies on eye-gaze and arrow cues. In Experiment 1, stronger attentional orienting was observed under the object-target condition compared with the scrambled-display condition, irrespective of whether gaze or arrow cues were used. The results indicated that meaningful targets produced stronger attentional orienting than did meaningless targets, regardless of the social characteristics of the target. Experiment 2, which investigated whether attentional orienting was influenced by differences in the
\end{abstract}

Tianyi Yan and Shuo Zhao are first coauthors on this work.

Shuo Zhao

zhaoshuo09@gmail.com

1 School of Life Science, Beijing Institute of Technology, Beijing, People's Republic of China

2 Key Laboratory of Convergence Medical Engineering System and Healthcare Technology, The Ministry of Industry and Information Technology, Beijing Institute of Technology, Beijing, China

3 Faculty of Human Health Science, Graduate School of Medicine, Kyoto University, 53 Shogoin Kawahara-cho, Sakyo-ku, Kyoto 606-8507, Japan

4 International Research Fellow of the Japan Society for the Promotion of Science, Tokyo, Japan

5 Organization for Promoting Neurodevelopmental Disorder Research, Kyoto, Japan

6 Department of Neurodevelopmental Psychiatry, Habilitation and Rehabilitation, Graduate School of Medicine, Kyoto University, Kyoto, Japan meaningfulness of targets regardless of their perceptual features, used participants' own faces and the faces of others as target stimuli; one's own face is typically more meaningful than the face of another. The results showed stronger attentional orienting in response to one's own face than in response to another's face under both gaze and arrow conditions. These findings suggest that the use of task-irrelevant meaningful information as targets may be effective in enhancing attention, regardless of perceptual features.

Keywords Attentional orienting $\cdot$ Gaze $\cdot$ Arrow $\cdot$ Meaningful information $\cdot$ Self-relevant information

In our daily lives, we simultaneously encounter various types of information with features of varying levels of importance. Generally, the ability to focus on important information and inhibit attention to other information is vital. This process works rapidly and effectively if directional information is available in the environment. An extensive body of literature has demonstrated that humans automatically orient their attention in the direction of centrally presented cues, such as gazes and arrows. In these studies (for a review, see Birmingham \& Kingstone, 2009), a central uninformative cue was used to direct attention toward the right or left of a screen, and a target was presented afterward at either the cued or the opposite location. The response times (RTs) to detect a target were faster if it appeared in the location congruent with the cue rather than in the opposite direction (i.e., cueing effect). The ability to process directional cues (e.g., a conspecific's gaze) rapidly provides an advantage for survival and would therefore be favored during the evolutionary process (Emery, 2000).

The majority of studies have investigated how various types of directional cues (e.g., gazes and arrows) trigger 
attentional orienting differently (Marotta, Lupiáñez, \& Casagrande, 2012). Birmingham and Kingstone (2009) proposed the importance of the environment in which directional cues are embedded. Recently, researchers found that attentional orienting in response to directional cues is also influenced by other factors, such as context (Bayliss, Schuch, \& Tipper, 2010; Kuhn \& Tipples, 2011). Bayliss et al. (2010) showed that the gaze direction of happy faces was more effective than the gaze direction of disgusted faces for triggering attentional orienting to pleasant targets. A similar result was demonstrated by Kuhn and Tipples (2011), who found that fearful faces led to an enhanced gaze-cueing effect relative to happy faces only while searching for a threatening target. Additional studies (Zhao, Uono, Yoshimura, Kubota, \& Toichi, 2013; Zhao, Uono, Yoshimura, \& Toichi, 2014, 2015a, 2015b) showed that attentional orienting was influenced by target characteristics, irrespective of cue characteristics. Specifically, the cueing effect in response to gaze or arrow cues was enhanced when using social voice versus nonsocial tone targets. The study suggested that greater attentional orienting was caused by the social properties of the targets. It is possible that an understanding of the operation of this mechanism will suggest specific target material to ameliorate the impairment of joint attention in a clinical population, such as those with autism spectrum disorder (ASD). However, because the features of voices and tones vary widely, these data have not yielded a definitive conclusion regarding the mechanisms underlying attentional orienting and how it is influenced by target processing.

There is another perspective regarding the characteristics of targets that moderate attentional orienting. In real life, the existence of task-irrelevant meaningful information improves cognitive processes (e.g., executive accuracy, processing speed). Fernandes and Manios (2012) showed that participants recollected words embedded in a meaningful (intact face) background more accurately than words in a meaningless (scrambled face) background. With respect to attentional orienting, Bayliss, Pellegrino, and Tipper (2005) found that among participants with low levels of autism-like traits, a stronger cueing effect occurred when the target appeared on an object (i.e., a face or tool) than when it appeared on a scrambled display, whereas the opposite result was found among participants with high levels of autism-like traits. Although these studies suggest that meaningful information in the environment enhances attentional orienting, the role of targets was not directly examined.

The aim of these experiment was to investigate the mechanisms underlying the influence of target processing on attentional orienting. Participants were asked to respond to an intact face (meaningful) and a scrambled face (meaningless) using both gaze and arrows as cues in Experiment 1A. In Experiment 1B, we examined attentional orienting as cued by gaze and arrows to compare targets corresponding to an intact house (meaningful) or a scrambled house (meaningless), with the aim of determining whether the pattern of attentional orienting obtained with face targets in Experiment $1 \mathrm{~A}$ would also be observed with house targets that were less socially relevant. The results of Experiments $1 \mathrm{~A}$ and $1 \mathrm{~B}$ showed that greater attention was accorded to the meaningful object (face or house) compared with the meaningless scrambled displays, irrespective of the social properties of the targets.

Furthermore, because there are perceptual as well as meaning-related differences between images of intact objects and scrambled displays, we manipulated attentional orienting by using two different meaningful stimuli as targets; these stimuli, tested in Experiment 2, elicited results that were consistent with Experiments 1A and 1B. Researchers (Lord \& Brown, 2004) have argued that an object or event attached to the self is more meaningful than one that is not (e.g., one's own face vs. that of another). Therefore, in Experiment 2, we explored whether attentional orienting was enhanced by a target corresponding to one's own face, which would presumably hold greater meaning, relative to the face of someone else.

\section{Experiment 1A}

\section{Method}

\section{Participants}

This research was approved by the local ethics committee of the School of Life Science at the Beijing Institute of Technology. No foreseeable risk to the participants was present, and personal identifying information was not collected. Participants provided written informed consent and background information. All procedures complied with the ethical standards of the 1964 Declaration of Helsinki regarding the treatment of human participants in research. Twenty-seven naïve individuals (mean age $=21.9 \pm 1.9 S D$ years; 15 men) participated in the study in exchange for 50 RMB. Twentyfour participants were right-handed (three participants were left-handed), as assessed by the Edinburgh Handedness Inventory (Oldfield, 1971). All participants had normal or corrected-to-normal visual and auditory acuity.

\section{Apparatus and stimuli}

Stimulus presentation and data acquisition were controlled by Presentation (Neurobehavioral Systems) on a Windows computer. Stimuli were presented on a 17 -inch 
CRT monitor (Dell: screen resolution $1,024 \times 768$ pixels; refresh rate $60 \mathrm{~Hz}$ ). The distance between the monitor and the participants was fixed at approximately $57 \mathrm{~cm}$ using a headrest.

The face stimuli were obtained from Ekman and Friesen (1976). The gaze-cue block (see Fig. 1a) used a photograph of a female model with a neutral face (MO). The gaze direction was then manipulated as a cue. The irises and pupils of the eyes were cut from the original photograph and pasted to fit over 18 pixels for the right or left side of the eyes using Photoshop (Adobe). We cropped the photographs in a rectangle $5.8^{\circ} \mathrm{cm}$ in width and $8.3^{\circ}$ in height. Pupil size was approximately $0.34^{\circ} \times 0.34^{\circ}$. In the arrow-cue block (see Fig. 1b), a symmetrical arrow was presented as a cue; an arrowhead appeared at one end with a tail at the opposite end. The arrow measured $5.8^{\circ}$ in width by $2.5^{\circ}$ in height and was light gray.

In addition, another female model with a neutral face (PF) was used as a target, with an intact face and a scrambled face, measuring $5.8^{\circ}$ in width $\times 8.3^{\circ}$ in height (see Fig. 1). The face (PF) was divided into several parts and randomly reorganized using Photoshop (Adobe) to create the scrambled face. The center of the target stimuli appeared at $9.95^{\circ}$ to the right or the left of the cue.

\section{Design and procedure}

As a within-subjects factor, the cueing paradigms (gaze or arrow as cue) were tested separately in the two types of blocks. In each cue block, two types of target objects (faces or scrambled face parts as targets) were separated into different blocks. In all experiments, the order of the four pattern blocks ( 2 Types of Cue $\times 2$ Types of Targets) was counterbalanced across participants (e.g., gaze cue-intact face, arrow cue-intact face, gaze cue-scrambled face, arrow cue-scrambled face). Thus, there were two types of cue: gazes and arrows. There were also two types of target objects: an intact face ("face") and a scrambled face. In valid trials, the direction of the cue was congruent with the target location; in invalid trials, the direction of the cue was incongruent with the target location.

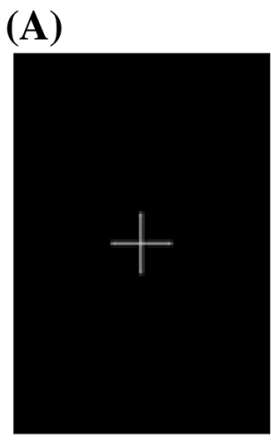

Fixation

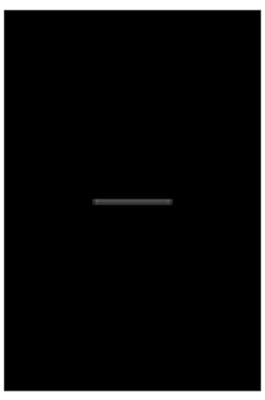

Background

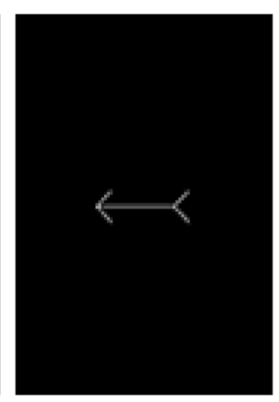

SOA

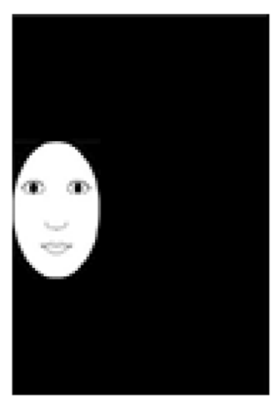

Target
$600 \mathrm{~ms}$

(B)

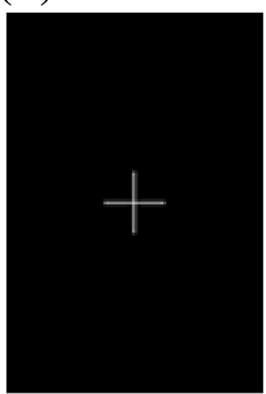

Fixation

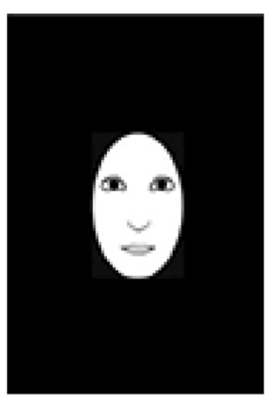

Background

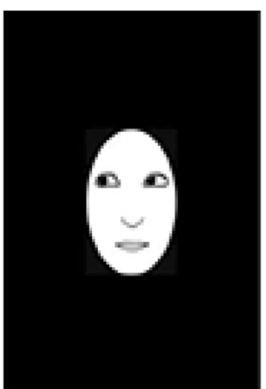

SOA

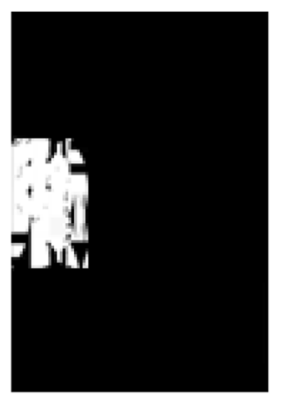

Target

$600 \mathrm{~ms}$

$500 \mathrm{~ms}$

$200 \mathrm{~ms}$

$300 \mathrm{~ms}$

Fig. 1 Illustration of the time course of conditions in Experiment 1. We used arrows (a) and gaze (b) stimuli as cues. The target stimuli corresponded either to an intact face or a scrambled face. Actual stimuli were photographs of faces (see Fig. 1 in Ekman \& Friesen, 1976) 
The sequence of stimulus presentation is shown in Fig. 1. In each trial, a fixation cross was first presented at the center of the screen for $600 \mathrm{~ms}$. A straight eye gaze (gaze block) or a transverse line (arrow block) was then presented at the location. After $500 \mathrm{~ms}$, a cue stimulus (i.e., gaze or arrow) oriented toward the right or the left was presented at the center of the screen. The stimulus onset asynchrony (SOA) between the target and the cue was $200 \mathrm{~ms}$. Subsequently, a target object (face or scrambled face) was presented at the left or right side of the screen for $300 \mathrm{~ms}$. To avoid any differential influence of disengagement from gaze and arrow cues, in this study we used an experimental design comparable with previous studies (Brignani, Guzzon, Marzi, \& Miniussi, 2009; Guzzon, Brignani, Miniussi, \& Marzi, 2010) whereby the gaze or arrow cue was removed before a target stimulus appeared on the display. The experiment was designed as a localization task. Participants were asked to indicate the target location (right or left side) as quickly and accurately as possible by pressing the corresponding key on the switch keypad using their dominant index or middle figure. Response times (RTs) were measured in each trial. The targets appeared on the same or the opposite side as the cue (gaze or arrow) direction. The target appeared at the cued location in $50 \%$ of the trials. The participants were told that the cue did not predict the target location and were instructed to fixate on the center of the screen in each trial.

The experiment consisted of eight blocks of 52 trials, including 24 catch trials in which the target did not appear. Forty-eight trials were performed under each condition. Participants were allowed to rest between blocks. Fifty-two practice trials preceded the experimental trials.

\section{Data analysis}

The data were analyzed using SPSS software. Incorrect responses ( $2.6 \%$ of the trials) and responses of $<150 \mathrm{~ms}$ or $>1,000 \mathrm{~ms}(0.22 \%$ of the trials $)$ were excluded from the RT analysis. The mean RT under each condition was calculated for each participant. The mean RTs were analyzed by two-way analysis of variance (ANOVA) with cue condition (gaze, arrow), target condition (face, scrambled face), and cue validity (valid, invalid) as the within-subjects factors.

\section{Results and discussion}

The data pertaining to errors revealed only a significant main effect of cue validity, $F(1,26)=18.7, p<.001$, partial $\eta^{2}=$ 0.42 , with fewer incorrect responses in the valid condition compared with the invalid condition ( 0.55 vs. $1.91 \%$; see Table 1a).
Table 1 Mean reaction times, standard deviations, and percentages of errors (\%) as functions of cue, target, and validity

\begin{tabular}{llllllll}
\hline Cue & Validity & Target & & \multicolumn{5}{c}{ Scrambled face } \\
& & Face & & & \\
\multirow{4}{*}{ Gaze } & & $M$ & $S D$ & $\% \mathrm{E}$ & $M$ & $S D$ & $\% \mathrm{E}$ \\
& Valid & 310.8 & 39.3 & 1.54 & 312.0 & 46.1 & 1.00 \\
& Invalid & 372.3 & 45.1 & 3.63 & 363.2 & 44.6 & 3.63 \\
Arrow & Valid & 313.1 & 43.0 & 1.08 & 319.0 & 50.0 & 0.93 \\
& Invalid & 375.3 & 44.1 & 4.63 & 373.4 & 44.3 & 4.01 \\
Cue & Validity & Target & & & & & \\
& & House & & & Scrambled house \\
& & $M$ & $S D$ & $\% \mathrm{E}$ & $M$ & $S D$ & $\% \mathrm{E}$ \\
\multirow{2}{*}{ Gaze } & Valid & 290.2 & 34.1 & 1.41 & 297.1 & 38.4 & 0.97 \\
& Invalid & 333.7 & 37.1 & 2.83 & 336.2 & 36.9 & 1.86 \\
\multirow{2}{*}{ Arrow } & Valid & 284.5 & 39.5 & 0.60 & 287.7 & 37.3 & 1.12 \\
& Invalid & 342.7 & 38.1 & 3.20 & 340.5 & 37.8 & 3.42 \\
\hline
\end{tabular}

We conducted a 2 (cue: gaze, arrow) $\times 2$ (target: face, scrambled face) $\times 2$ (validity: valid, invalid) repeatedmeasures ANOVA on these RTs (see Table 1a). A main effect of validity was significant, $F(1,26)=466.7, p<$ .001 , partial $\eta^{2}=0.95$, with faster responses under the valid condition versus the invalid one $(313 \mathrm{~ms}$ vs. 371 $\mathrm{ms})$. However, no significant main effect was detected for cue, $F(1,26)=0.60, p=.44$, partial $\eta^{2}=0.02$, or target, $F(1,26)=0.25, p=.62$, partial $\eta^{2}=0.01$. It is important to note that a significant interaction of Target $\times$ Validity was found, $F(1,26)=9.06, p=.006$, partial $\eta^{2}=0.26$, but no significant interaction was detected for Cue $\times$ Validity, $F(1,26)=0.34, p=.56$, partial $\eta^{2}=$ 0.13 , or Cue $\times$ Target $\times$ Validity, $F(1,26)=0.21, p=$ .65 , partial $\eta^{2}=0.01$.

A post hoc test revealed a significant difference between face and scrambled-face targets under the invalid condition $(p$ $=.01)$, with slower responses to face versus scrambled-face targets ( $374 \mathrm{~ms}$ vs. $368 \mathrm{~ms}$ ). No difference was found between

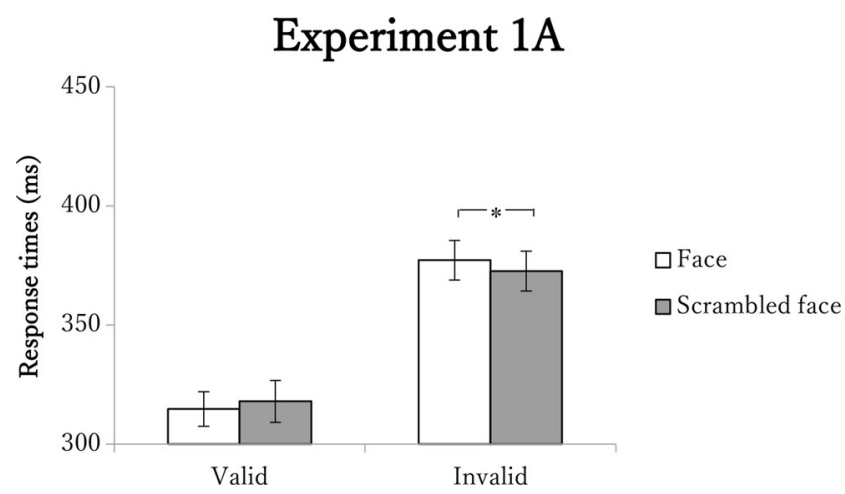

Fig. 2 Mean (with $S E$ ) RTs under the face and scrambled-face target conditions in Experiment 1A. Error bars represent standard errors. * $p<.05$ 
face and scrambled-face targets (312 ms vs. $315 \mathrm{~ms}$ ) under the valid condition $(p=.23)$. The results indicated that participants were more strongly triggered to respond with attentional orienting to targets corresponding to faces than they were to scrambled faces (see Fig. 2).

\section{Experiment 1B}

In Experiment 1B, we used images of a house and a scrambled house as targets in an attentional-orienting task involving responses to arrow and gaze cues, with the aim of determining whether the enhancement of attentional orienting would occur even when nonsocially meaningful targets were used.

\section{Method}

\section{Participants}

Twenty-eight naïve participants (mean age $=21.4 \pm 2.0 \mathrm{SD}$ years; 15 men) participated in the study for payment. Twenty- four participants were right-handed and four were left-handed, as assessed by the Edinburgh Handedness Inventory (Oldfield, 1971), and all participants had normal or corrected-to-normal visual and auditory acuity (see Fig. 3).

Apparatus, stimuli, design, procedure, and analysis

All conditions were the same as in Experiment 1A, except that the target stimulus in this experiment corresponded to a house or a scrambled house. The house images were taken from a previous study (Wang et al., 2013). Incorrect responses (1.6\% of the trials $)$ and responses of $<150 \mathrm{~ms}$ or $>1,000 \mathrm{~ms}(0.13 \%$ of the trials) were excluded from the RT analysis.

\section{Results and discussion}

The error data revealed only a significant main effect of validity, $F(1,27)=12.1, p=.002$, partial $\eta^{2}=0.31$, with fewer incorrect responses under the valid versus the invalid condition ( 0.49 vs. $1.36 \%$; see Table $1 b)$.

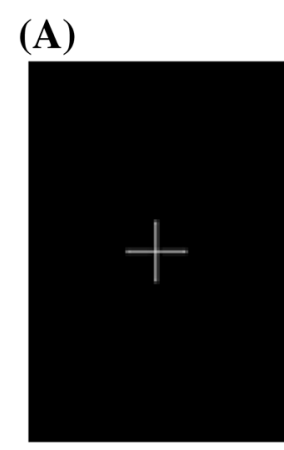

Fixation

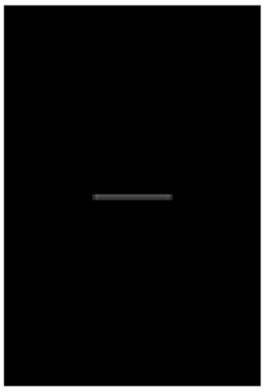

Background

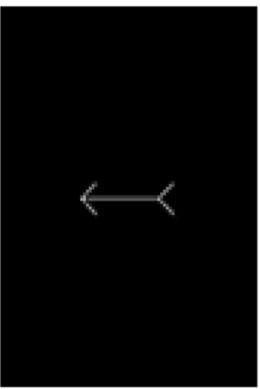

SOA

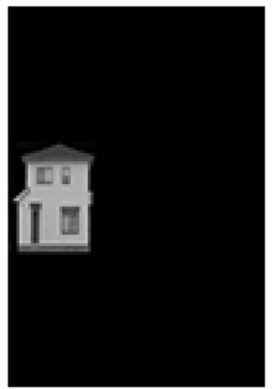

Target

$\begin{array}{llll}\longrightarrow & \\ & 500 \mathrm{~ms} & 200 \mathrm{~ms} & 300 \mathrm{~ms}\end{array}$

(B)
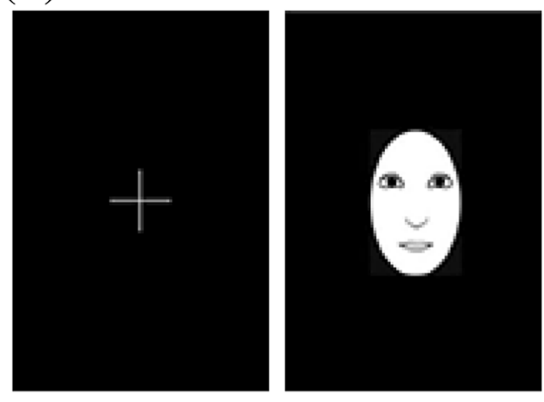

Fixation

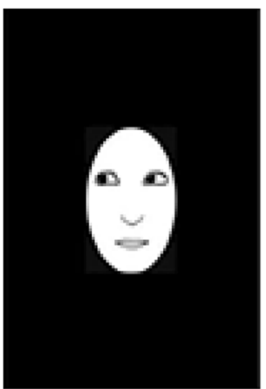

SOA

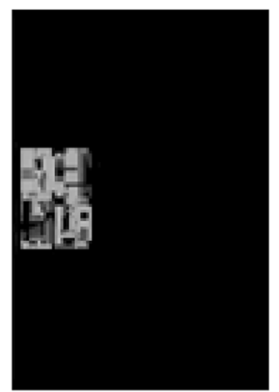

Target

$600 \mathrm{~ms}$

$500 \mathrm{~ms}$

$200 \mathrm{~ms}$

$300 \mathrm{~ms}$

Fig. 3 Illustration of the time course of conditions in Experiment 1B. We used arrow (a) and gaze (b) stimuli as cues. The target stimulus was either an intact house (hereafter, "house") or a scrambled house 
We conducted a 2 (cue: gaze, arrow) $\times 2$ (target: house, scrambled house) $\times 2$ (validity: valid, invalid) repeated-measures ANOVA on these RTs (see Table 1b). The main effect of validity was significant, $F(1,27)=331.4, p<.001$, partial $\eta^{2}=0.93$, with faster responses under the valid versus the invalid condition (290 ms vs. $338 \mathrm{~ms}$ ). However, no significant main effects of cue, $F(1,27)=0.02, p=.89$, partial $\eta^{2}=0.001$, or target, $F(1$, 27) $=0.38, p=.06$, partial $\eta^{2}=0.12$, were observed. A significant interaction of Cue $\times$ Validity was also found, $F(1,27)=12.1, p=$ .002 , partial $\eta^{2}=0.31$. However, a post hoc test revealed no significant difference between gaze and arrow cues under either the valid or invalid condition (both $p \mathrm{~s}>.05$ ). In addition, no significant interaction was detected for Cue $\times$ Target $\times$ Validity, $F(1,27)=0.02, p=.88$, partial $\eta^{2}=0.001$.

A significant interaction of Target $\times$ Validity was found, $F(1,27)=7.31, p=.01$, partial $\eta^{2}=0.21$, and a post hoc test revealed a significant difference between house and scrambled house targets under the valid condition $(p=.04)$, with faster responses to house than to scrambled-house targets $(287 \mathrm{~ms}$ vs. $292 \mathrm{~ms}$ ); no difference was found between house and scrambled-house targets ( $338 \mathrm{~ms}$ vs. $338 \mathrm{~ms}$ ) under the invalid condition $(p=.89)$. The results indicated enhanced attentional orienting when house versus scrambled-house targets were used (see Fig. 4).

We found comparable patterns of attentional orienting across Experiments $1 \mathrm{~A}$ and $1 \mathrm{~B}$, both of which used gaze and arrow cues in an environment in which the cue and target were fixed in a block. That is, stronger attentional orienting was shown for intact objects (faces or houses) relative to scrambled-target conditions, irrespective of cue. Based on these findings, we suggest that attentional orienting is enhanced by meaningful targets, regardless of whether they are social.

\section{Experiment 2}

Experiment 1 demonstrated that the existence of a meaningful target moderates attentional orienting triggered by gaze or

\section{Experiment 1B}

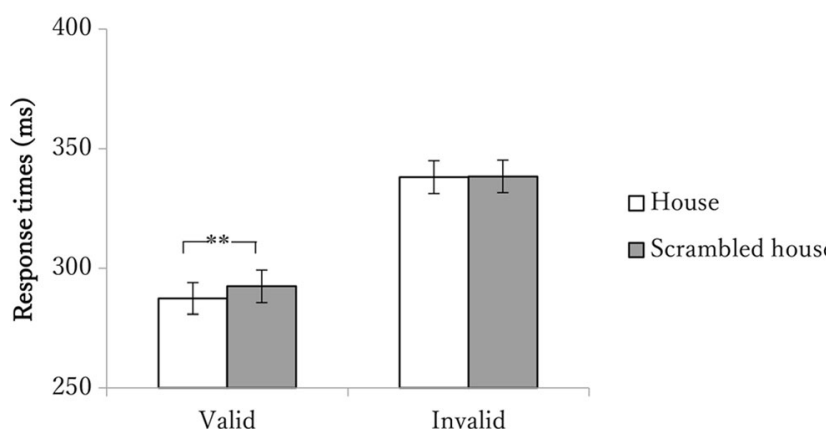

Fig. 4 Mean (with $S E$ ) RTs under the house and scrambled-house target conditions in Experiment 1B. Error bars represent standard errors. $* * p<.01$ arrows. However, objects and scrambled displays have different perceptual features. Thus, the results of Experiment 1 did not clearly reveal whether attentional orienting was triggered by the meaningfulness or by the perceptual features of a target. In Experiment 2, we investigated whether attentional orienting was influenced by differences in the meaningfulness of targets, regardless of perceptual features. Hence, we manipulated own- and other-face targets under the assumption that despite the similarity of their perceptual features, the former are typically more meaningful than the latter. We hypothesized that attentional orienting would be enhanced by the own-face target relative to the other-face target.

\section{Method}

\section{Participants}

Twenty-seven naïve participants (mean age $=20.7 \pm 1.1 S D$ years; 15 men) participated in the study for payment. Twentythree participants were right-handed and four were left-handed, as assessed by the Edinburgh Handedness Inventory (Oldfield, 1971), and all participants had normal or corrected-to-normal visual and auditory acuity.

\section{Apparatus, stimuli, design, procedure, and analysis}

All conditions were the same as in Experiment 1A, except that the target stimuli (i.e., face and scrambled face) were replaced by the participant's own face and the face of someone else, respectively. The order of all blocks was counterbalanced across participants (e.g., gaze cue-own face, arrow cue-own face, gaze cue-other face, arrow cue-other face). Photos of participants were taken immediately before the experiment (participants did not see these pictures before the experiment), whereas photos of unknown people (a female and a male) were taken before the study. All face images were photos taken under the same conditions and then converted into a black-and-white background, and the same size, using Photoshop CS6 (Adobe). Moreover, the luminance of the images was measured using MATLAB 2012 (MathWorks). The degree of luminance was not different between own and other faces (one-sample $t$ test), $t(26)=0.348, p=.731$. Before the experiment, all participants confirmed that they did not know the persons used in the photos of someone else. Incorrect responses $(0.9 \%$ of the trials) and responses of $<150 \mathrm{~ms}$ or $>1,000 \mathrm{~ms}$ ( $0.15 \%$ of the trials) were excluded from the RT analysis.

\section{Results and discussion}

The data on errors did not reveal any significant main effect or interaction effect (all $p \mathrm{~s}>.05$ ). We conducted a 2 (cue: gaze, arrow) $\times 2$ (target: own face, other face) $\times 2$ (validity: valid, 
Table 2 Mean reaction times, standard deviations, and percentages of errors $(\mathrm{E} \%)$ as functions of cue, target, and validity

\begin{tabular}{|c|c|c|c|c|c|c|c|}
\hline \multirow[t]{3}{*}{ Cue } & \multirow[t]{3}{*}{ Validity } & \multicolumn{6}{|l|}{ Target } \\
\hline & & \multicolumn{3}{|c|}{ Own face } & \multicolumn{3}{|c|}{ Other face } \\
\hline & & $M$ & $S D$ & $\% \mathrm{E}$ & $M$ & $S D$ & $\% \mathrm{E}$ \\
\hline \multirow[t]{2}{*}{ Gaze } & Valid & 331.7 & 47.1 & 1.00 & 335.4 & 40.6 & 1.16 \\
\hline & Invalid & 386.7 & 54.2 & 1.31 & 386.1 & 48.6 & 1.16 \\
\hline \multirow[t]{2}{*}{ Arrow } & Valid & 328.2 & 42.0 & 0.39 & 331.6 & 41.0 & 0.85 \\
\hline & Invalid & 385.1 & 47.4 & 1.31 & 375.7 & 43.7 & 0.93 \\
\hline
\end{tabular}

invalid) repeated-measures ANOVA on these RTs (see Table 2). The main effect of validity was significant, $F(1$, 26) $=405.5, p<.001$, partial $\eta^{2}=0.94$, with faster responses under the valid versus the invalid condition $(331 \mathrm{~ms}$ vs. 383 $\mathrm{ms})$. However, there were no significant main effects of cue, $F(1,26)=1.21, p=0.28$, partial $\eta^{2}=0.04$, or target, $F(1,26)=$ $0.18, p=.67$, partial $\eta^{2}=0.07$. It is important to note that a significant interaction of Target $\times$ Validity was found, $F(1,26)$ $=9.9, p=.004$, partial $\eta^{2}=0.28$, but no significant interaction was detected for Cue $\times$ Validity, $F(1,26)=0.25, p=.62$, partial $\eta^{2}=0.01$, or Cue $\times$ Target $\times$ Validity, $F(1,26)=$ $0.26, p=.12$, partial $\eta^{2}=0.09$.

A post hoc test revealed a significant difference between own- and other-face targets under the invalid condition ( $p=$ $.04)$, with slower responses to own-face than to other-face targets ( $385 \mathrm{~ms}$ vs. $380 \mathrm{~ms}$ ); no difference was found between own-face and other-face targets ( $333 \mathrm{~ms}$ vs. $330 \mathrm{~ms}$ ) under the valid condition ( $p=0.1$; see Fig. 5).

These results indicated that attentional orienting was enhanced when using own-face versus other-face targets. It is common for people to have a well-established self-schema that facilitates the encoding and retrieval of information related to the self, which is more meaningful than that related to others (Lord \& Brown, 2004; Maki \& McCaul, 1985; Rogers, Kuiper, \& Kirker, 1977). Consistent with these findings, we

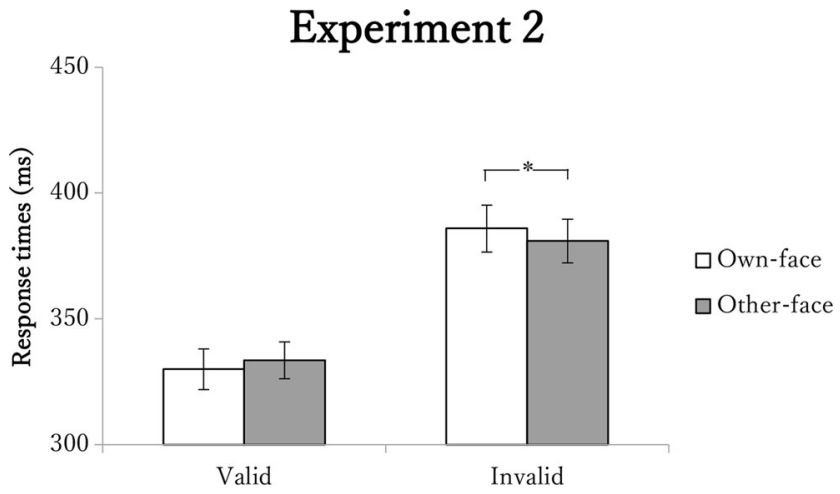

Fig. 5 Mean (with $S E$ ) RT differences between invalid and valid conditions for own-face and other-face stimuli. Error bars represent standard errors. $* p<.05$ found that attentional orienting was enhanced for each individual by the presence of own-face targets, which involved more meaningful information than other-face targets. Therefore, the findings demonstrated that attentional orienting is enhanced by self-relevant meaningful targets.

\section{Combined analysis of Experiments 1 and 2}

To evaluate the different pattern of attentional orienting in response to face and house targets between valid and invalid conditions, the results of Experiments 1 and 2 were compared directly using a four-way repeated-measures ANOVA on RTs, with Cue (gaze, arrow), Target (meaningful, less meaningful objects) and Validity (valid, invalid) as within-participant factors, and Experiment (1A, 1B, 2) as the between-participant factor. An interaction of Target $\times$ Validity was seen, $F(1,79)=$ 25.96, $p<.001$, partial $\eta^{2}=0.25$, but no other factor (Cue, Experiment) showed a significant interaction with Target $x$ Validity (both $p s>0.1$ ). A post hoc test revealed a significant difference between targets under the valid condition ( $p=$ .003 ), with faster responses to meaningful than to less meaningful objects ( $309 \mathrm{~ms}$ vs. $314 \mathrm{~ms}$ ), and the invalid condition $(p=.004)$ with slower responses to meaningful than to less meaningful objects (366 ms. vs. $362 \mathrm{~ms}$ ). This analysis demonstrates that the modulation of Validity $\times$ Target was consistent across Experiments 1 and 2. That is, attentional orienting was enhanced by meaningful targets under both valid and invalid conditions.

\section{General discussion}

The two experiments revealed the critical role played by target processing in attentional orienting using eye-gaze and arrow cues. In Experiment 1, more robust attentional orienting was triggered when targets were meaningful objects (a social face or a nonsocial house) than when they were meaningless, scrambled displays, irrespective of the specific cue stimulus used. In Experiment 2, we investigated whether attentional orienting was influenced by differences in the meaningfulness of targets, despite comparable perceptual features between targets. We found stronger attentional orienting in response to own-face than to other-face targets.

Previous studies have also reported the influence of target objects on attentional orienting based on gaze and arrow cues (Bayliss \& Tipper, 2005; Zhao et al., 2013; Zhao et al., 2014, 2015a, 2015b). One might argue that enhanced attentional capture by a meaningful target (e.g., faces, houses, own faces) offers a simple explanation for enhanced attentional orienting. However, these results did not find enhanced RTs to these targets across experiments. Specifically, in Experiment 1A, the enhanced attentional orienting originated from the RT delay to face targets under the invalid condition. These findings 
indicate that attentional capture by targets and attentional orienting to the cued location did not have an additive effect in this study. The processing of target characteristics moderated the processing of gaze and arrow cues. Most previous studies (Bayliss et al., 2005; Marotta et al., 2013; Senju, Tojo, Dairoku, \& Hasegawa, 2004; Uono, Sato, \& Toichi, 2009; see a review by Frischen, Bayliss, \& Tipper, 2007) have focused on the importance of cue characteristics and observer traits in attentional orienting. However, this study suggests the importance of external factors other than the cue-observer relationship for completely understanding attentional orienting in daily situations.

This study also provides information relevant to understanding the types of target that effectively enhance attentional orienting using centrally presented cues. Previous studies (Zhao et al., 2013; Zhao et al., 2014, 2015a, 2015b) have proposed that attentional orienting is influenced only if the target is social (i.e., enhanced orienting is triggered by voice compared with tone targets). Our results showed stronger attentional orienting with face (meaningful social stimuli) compared with scrambled-face (meaningless nonsocial stimuli) targets in Experiment 1A. The same pattern of results was also observed with house (meaningful nonsocial stimuli) compared with scrambled-house (meaningless nonsocial stimuli) targets in Experiment 1B. These results extend the findings of a previous study (Bayliss \& Tipper, 2005) in which attentional orienting was facilitated when the target appeared in a context with recognizable objects rather than in a scrambled context, regardless of social context. Consistent with the previous study (Bayliss \& Tipper, 2005), we demonstrated that attentional orienting was also influenced by a meaningful target, regardless of whether the stimulus was social in nature.

Moreover, the results of Experiment 2 suggest that meaningful targets in the form of self-relevant stimuli are more effective than stimuli related to others in enhancing attentional orienting, as stronger attentional orienting was shown under the own-face versus the other-face target condition, irrespective of cue. Previous studies have proposed that cues with selfrelevant information are processed more effectively than those with other-relevant information with regard to spatial attention (Alexopoulos, Muller, Ric, \& Marendaz, 2012; Hunger \& Hunt, 2012). For instance, Hunger and Hunt (2012) showed that the effect of gaze cues on target detection increased for faces morphed on the basis of the participants' face. Other studies (Sui, Liu, Wang, \& Han, 2009) have also shown that attentional orienting was enhanced when self-relevant versus other-relevant stimuli were used as cues. Our study extends these studies by demonstrating that the self-relevance of targets plays an important role in attentional orienting triggered by centrally presented cues. Additionally, in real life, it seems likely that people would have a strong tendency to be interested in themselves and in matters that concern them (Maki \& McCaul, 1985; Rogers et al., 1977). Previous studies have revealed that trained individuals with $\mathrm{ASD}$, even with impaired joint attention, respond to and initiate joint attention when they are engaged with material that is of interest to them (Kryzak, Bauer, Jones, \& Sturmey, 2013; Naoi, Tsuchiya, Yamamoto, \& Nakamura, 2008). Because people are typically interested in different objects, the self-relevance of these objects varies greatly across individuals. We suggest that different attentional orienting patterns can be triggered by different target objects in different individuals; thus, such variance might affect social orienting in the development of social cognition. For example, the atypical social orienting of individuals with ASD may be influenced by their interests.

Taken together, the results of both experiments reveal comparable patterns of attentional orienting between gaze and arrow cues. Previous studies (Birmingham, \& Kingstone, 2009; Zhao et al., 2014) have proposed that differences between gaze and arrow cues may become apparent only when they are embedded within a rich environment, but that any differences are negligible in a simple environment. Consistent with this notion, our results indicate that arrow cues produce a shift in attentional orienting that is similar to that produced by gaze cues if the cue and the target are fixed in each block (i.e., representing a simple environment). Considering that the natural environment is very complex, future studies should investigate whether target processing affects attentional orienting differently when gaze and arrow cues are used if such cues are randomly presented in a block (i.e., in a rich environment).

Furthermore, to evaluate the pattern of attentional orienting across experiments, a combined analysis was made of Experiments 1 and 2. The results showed that stronger attention orienting was triggered by gaze and arrow cues in both valid and invalid conditions when presenting a meaningful target rather than one of less meaning. A facilitated orienting to meaningful versus less meaningful targets was observed under the valid condition, whereas under the invalid condition, there was a slower disengagement from cued locations when meaningful versus less meaningful targets were used. We propose a possible mechanism that attention orienting by gaze and arrows between targets might be modulated by the context of the attentional shift. The current task manipulated the type of target separately in each block. Participants were completely certain about the identity of the future target in a specific block. They could implicitly evaluate the importance of their environment and bias the processing of cues in the environment. For example, when two people are in a dark and dangerous environment (i.e., a highly important condition), one person's eye gaze is critical for the other because it can help him or her to better and rapidly avoid a suddenly dangerous target. Through the other's eye gaze, one person can rapidly capture a target being looked at, but it can also be difficult to disengage from it even, when a target appears at an opposite location. In the current task, given that the importance of the environment would be raised when presenting a target that is 
more meaningful than less meaningful, participants' attention could be biased to the processing of cues and be strongly triggered by cues in the meaningful target block. To examine this proposition, further research should investigate the effect of environmental conditions between target contexts during attentional orienting - that is, manipulating attentional orienting between target meaningfulness is blocked (as in a simple environment) and varies randomly across trials (as in a rich environment). Another possible explanation might be that the target processing moderates online attentional processing. Previous studies have proposed that the subcortical regions, such as the amygdala, rapidly process objects prior to the visual cortices (within $200 \mathrm{~ms}$; e.g., Sato et al., 2011) and moderate the activity of other brain regions depending on their emotional meaning (e.g., Vuilleumier \& Pourtois, 2007). In the current task, the target appeared at $200 \mathrm{~ms}$ after cue onset. The time window of the processing in the subcortical regions could overlap with that of orienting and holding attention to the cued location because previous electrophysiological studies have shown that attention orienting by gaze and arrows occur 200-400 ms after cue onset (e.g., Uono, Sato, \& Kochiyama, 2014). Thus, the rapid processing of a meaningful target might enhance the activity of the attentional system under the $200 \mathrm{~ms}$ SOA condition but not a longer SOA condition ( $\sim 400 \mathrm{~ms}$ ). Investigations into the SOA condition, combined with electroencephalogram or magnetoencephalography, both of which have very high temporal resolution, could be informative for online attentional processing in future research. Although our data do not allow any definite conclusion to be drawn about the underlying mechanism for the effect of the target processing, they suggest that the attentional system triggered by gaze and arrows works flexibly depending on the environment over short or long time scales and that the attentional advantage of the target effect $(\sim 10 \mathrm{~ms})$ would allow us to adapt to a changing environment.

The purpose of this study was to investigate the mechanisms underlying the influence of target processing on attentional orienting triggered by gaze and arrows. Equivalent patterns of attentional orienting were observed in response to gaze and arrow cues. The results of Experiment 1 showed that attentional orienting was enhanced by a meaningful target, regardless of whether its meaning was social. Furthermore, the use of self-relevant stimuli as meaningful targets, involving perceptual features that were comparable to less meaningful other-relevant stimuli, resulted in enhanced attentional orienting, as demonstrated by a stronger attentional orienting effect in response to own-face targets relative to other-face targets. Because the self-relevance of specific objects varies greatly across individuals, different attentional orienting patterns may be induced by the same target objects. Further studies along these lines may provide a new perspective with which to understand differences in human attentional orienting in daily situations.
Acknowledgments We acknowledge and thank the subjects involved in the study. This study was supported by a Grant-in-Aid for Overseas JSPS Fellows (15F15307), the National Natural Science Foundation of China (Grant Nos. 61305142), and the Organization for Promoting Developmental Disorder Research.

Author contributions $\quad$ S. Z. and T. Y. designed the experiment. A. T. collected and analyzed the data. S. Z., X. B., and A. T. prepared the figures. S. Z., T. Y., S. U., S. Y., and M. T. wrote the manuscript.

\section{References}

Alexopoulos, T., Muller, D., Ric, F., \& Marendaz, C. (2012). I, me, mine: Automatic attentional capture by self-related stimuli. European Journal of Social Psychology, 42, 770-779.

Bayliss, A. P., Pellegrino, G. D., \& Tipper, S. P. (2005). Sex differences in eye gaze and symbolic cueing of attention. The Quarterly Journal of Experimental Psychology Section A, 58, 631-650.

Bayliss, A. P., Schuch, S., \& Tipper, S. P. (2010). Gaze cueing elicited by emotional faces is influenced by affective context. Visual Cognition, $18,1214-1232$.

Bayliss, A. P., \& Tipper, S. P. (2005). Gaze and arrow cueing of attention reveals individual differences along the autism spectrum as a function of target context. British Journal of Psychology, 96, 95-114.

Birmingham, E., \& Kingstone, A. (2009). Human social attention. Annals of the New York Academy of Sciences, 1156, 118-140.

Brignani, D., Guzzon, D., Marzi, C. A., \& Miniussi, C. (2009). Attentional orienting induced by arrows and eye-gaze compared with an endogenous cue. Neuropsychologia, 47, 370-381.

Ekman, P., \& Friesen, W. V. (1976). Pictures of facial affect. Palo Alto: Consulting Psychologists Press.

Emery, N. J. (2000). The eyes have it: The neuroethology, function and evolution of social gaze. Neuroscience \& Biobehavioral Reviews, 24, 581-604.

Fernandes, M. A., \& Manios, M. (2012). How does encoding context affect memory in younger and older adults? The Quarterly Journal of Experimental Psychology, 65, 1699-1720.

Frischen, A., Bayliss, A. P., \& Tipper, S. P. (2007). Gaze cueing of attention: Visual attention, social cognition, and individual differences. Psychological Bulletin, 133, 694-724.

Guzzon, D., Brignani, D., Miniussi, C., \& Marzi, C. A. (2010). Orienting of attention with eye and arrow cues and the effect of overtraining. Acta Psychologica, 134, 353-362.

Hunger, C. J., \& Hunt, A. R. (2012). Physical self-similarity enhances the gaze-cueing effect. The Quarterly Journal of Experimental Psychology, 65, 1250-1259.

Kryzak, L. A., Bauer, S., Jones, E. A., \& Sturmey, P. (2013). Increasing responding to others' joint attention directives using circumscribed interests. Journal of Applied Behavior Analysis, 46, 674-679.

Kuhn, G., \& Tipples, J. (2011). Increased gaze following for fearful faces: It depends on what you're looking for! Psychonomic Bulletin and Review, 18, 89-95.

Lord, R. G., \& Brown, D. J. (2004). The working self-concept and behavior. In A. P. Brief, J. P. Walsh, P. C. Early, \& S. L. Rynes (Eds.), Leadership processes and follower self-identity (pp. 12-32). Mahwah: Erlbaum.

Maki, R. H., \& McCaul, K. D. (1985). The effects of self-reference versus other reference on the recall of traits and nouns. Bulletin of the Psychonomic Society, 23, 169-172.

Marotta, A., Lupiáñez, J., \& Casagrande, M. (2012). Investigating hemispheric lateralization of reflexive attention to gaze and arrow cues. Brain Cognition, 80, 361-366. 
Marotta, A., Pasini, A., Ruggiero, S., Maccari, L., Rosa, C., Lupiáñez, J., \& Casagrande, M. (2013). Inhibition of return in response to eye gaze and peripheral cues in young people with Asperger's syndrome. Journal of Autism and Developmental Disorders, 43, 917-923.

Naoi, N., Tsuchiya, R., Yamamoto, J.-I., \& Nakamura, K. (2008). Functional training for initiating joint attention in children with autism. Research in Developmental Disabilities, 29, 595-609.

Oldfield, R. C. (1971). The assessment and analysis of handedness: The Edinburgh Inventory. Neuropsychologia, 9, 97-113.

Rogers, T. B., Kuiper, N. A., \& Kirker, W. S. (1977). Self-reference and the encoding of personal information. Journal of Personality and Social Psychology, 35, 677-688.

Sato, W., Kochiyama, T., Uono, S., Matsuda, K., Usui, K., Inoue, Y., \& Toichi, M. (2011). Rapid amygdala gamma oscillations in response to eye gaze. PLoS ONE, 6, e28188. doi:10.1371/journal. pone. 0028188

Senju, A., Tojo, Y., Dairoku, H., \& Hasegawa, T. (2004). Reflexive orienting in response to eye gaze and an arrow in children with and without autism. Journal of Child Psychology and Psychiatry, $45,445-458$

Sui, J., Liu, C. H., Wang, L., \& Han, S. (2009). Attentional orientation induced by temporarily established self-referential cues. The Quarterly Journal of Experimental Psychology, 62, 844-849.

Uono, S., Sato, W., \& Kochiyama, T. (2014). Commonalities and differences in the spatiotemporal neural dynamics associated with automatic attentional shifts induced by gaze and arrows. Neuroscience Research, 87, 56-65.

Uono, S., Sato, W., \& Toichi, M. (2009). Dynamic fearful gaze does not enhance attentional orienting in individuals with Asperger's disorder. Brain Cognition, 71, 229-233.

Vuilleumier, P., \& Pourtois, G. (2007). Distributed and interactive brain mechanisms during emotion face perception: Evidence from functional neuroimaging. Neuropsychologia, 45, 174-194.

Wang, B., Yan, T., Wu, J., Chen, K., Imajyo, S., Ohno, S., \& Kanazawa, S. (2013). Regional neural response differences in the determination of faces or houses positioned in a wide visual field. PLOS ONE, 8 , e72728. doi:10.1371/journal.pone.0072728

Zhao, S., Uono, S., Yoshimura, S., Kubota, Y., \& Toichi, M. (2013). Can gaze-cueing be helpful for detecting sound in autism spectrum disorder? Research in Autism Spectrum Disorders, 7, 1250-1256.

Zhao, S., Uono, S., Yoshimura, S., \& Toichi, M. (2014). Attentional orienting by eye gaze and arrows reveals flexibility to environmental changes. Acta Psychologica, 150, 100-105.

Zhao, S., Uono, S., Yoshimura, S., \& Toichi, M. (2015a). Is impaired joint attention present in non-clinical individuals with high autistic traits? Molecular Autism, 6, 67. doi:10.1186/s13229-015-0059-3

Zhao, S., Uono, S., Yoshimura, S., \& Toichi, M. (2015b). Self make-up: The influence of self-referential processing on attention orienting. Scientific Report, 5, 14169. 